\title{
Intrapartum fetal heart rate monitoring using a handheld Doppler versus Pinard stethoscope: a randomized controlled study in Dar es Salaam
}

This article was published in the following Dove Press journal: International Journal of Women's Health

\author{
Benjamin A Kamala ${ }^{1,2}$ \\ Hussen L Kidanto ${ }^{3,4}$ \\ Peter J Wangwe ${ }^{2,5}$ \\ Ingvild Dalen ${ }^{3}$ \\ Estomih R Mduma ${ }^{3,6}$ \\ Jeffrey M Perlman ${ }^{7}$ \\ Hege L Ersdal ${ }^{1,8}$
}

'Faculty of Health Sciences, University of Stavanger, Stavanger, Norway;

${ }^{2}$ Department of Obstetrics and Gynecology, Muhimbili National Hospital, Dar es Salaam, Tanzania; ${ }^{3}$ Department of Research, Stavanger University Hospital, Stavanger, Norway; ${ }^{4}$ School of Medicine, Aga Khan University, Dar es Salaam, Tanzania; ${ }^{5}$ Department of Obstetrics and Gynecology, Muhimbili University of Health and Allied Science, Dar es Salaam, Tanzania; ${ }^{6}$ Department of Research, Haydon Lutheran Hospital, Manyara, Tanzania; ${ }^{7}$ Department of Pediatrics, Weill Medical College, New York, NY, USA; ${ }^{8}$ Department of Anesthesiology and Intensive Care, Stavanger University Hospital, Stavanger, Norway
Correspondence: Benjamin A Kamala Faculty of Health Sciences, University of Stavanger, Stavanger, Norway

Tel +474644 I 263

Email ba.kamala@stud.uis.no
Background: Fetal stethoscopes are mainly used for intermittent monitoring of fetal heart rate (FHR) during labor in low-income countries, where perinatal mortality is still high. Handheld Dopplers are rarely available and are dependent on batteries or electricity. The objective was to compare the Pinard stethoscope versus a new wind-up handheld Doppler in the detection of abnormal FHR.

Materials and methods: We conducted a randomized controlled study at Muhimbili National Hospital, Tanzania, from April 2013 to September 2015. Women with gestational age $\geq 37$ weeks, cephalic presentation, normal FHR on admission, and cervical dilatation $<7 \mathrm{~cm}$ were included. Primary outcome was abnormal FHR detection ( $<120$ or $>160$ beats $/ \mathrm{min}$ ). Secondary endpoints were time to delivery, mode of delivery, and perinatal outcomes. $\chi^{2}$, Fisher's exact test, MannWhitney test, and logistic regression were conducted. Unadjusted and adjusted odds ratios were calculated with respective $95 \%$ confidence interval.

Results: In total, 2,844 eligible women were assigned to FHR monitoring with Pinard $(n=1,423)$ or Doppler $(\mathrm{n}=1,421)$. Abnormal FHRs were more often detected in the Doppler $(6.0 \%)$ versus the Pinard (3.9\%) arm (adjusted odds ratio $=1.59,95 \%$ confidence interval: $1.13-2.26, p=0.008$ ). Median (interquartile range) time from abnormal FHR detection to delivery was comparable between Doppler and Pinard, ie, $80(60,161)$ and $89(52,165)$ minutes, respectively, as was the incidence of cesarean delivery $(12.0 \%$ versus $12.2 \%)$. The incidence of adverse perinatal outcomes (fresh stillbirths, 24-hour neonatal admissions, and deaths) was similar overall; however, among newborns with abnormal FHR delivered vaginally, adverse outcomes were less incident in Doppler ( 7 of 43 births, 16.3\%) than in the Pinard arm (10 of 23 births, 43.5\%), $p=0.021$.

Conclusion: Intermittent FHR monitoring using Doppler was associated with an increased detection of abnormal FHR compared to Pinard in a low-risk population. Time intervals from abnormal FHR detection to delivery were longer than recommended in both arms. Perinatal outcomes were better among vaginally delivered newborns with detected abnormal FHR in the Doppler arm.

Keywords: fetal heart rate, perinatal outcomes, Pinard stethoscope, Doppler

\section{Introduction}

Childbirth is regarded as a normal physiological process; however, in low-income countries (LIC) there is an increased risk of mortality for both the mother and her newborn. ${ }^{1}$ More than $99 \%$ of all newborn deaths occur in LIC, with important causes including lack of skilled personnel, essential technology, and supplies, including medicines. ${ }^{2}$ Annually, 1.02 million fresh stillbirths (FSB) occur, ${ }^{1,3,4}$ and intrapartumrelated neonatal deaths account for almost $40 \%$ of 2.6 million neonatal deaths. ${ }^{5}$ 
Intrapartum interruption of placental blood flow to the fetus has both short-term and long-term adverse consequences. Short-term outcomes include stillbirth, low Apgar score, need for resuscitations, neonatal intensive care unit admissions, and early neonatal deaths. ${ }^{6}$ Long-term outcomes include cognitive and behavioral disabilities - affecting perhaps as much as one million children each year. ${ }^{7,8} \mathrm{~A}$ focus on high coverage of good quality care during birth, including timely identification and rescue of the fetus from intrapartum hypoxia, ${ }^{9}$ will save the lives of many newborns. ${ }^{10}$ Effective fetal heart rate (FHR) monitoring tools for early detection of FHR abnormalities should facilitate appropriate obstetrical interventions, and hence contribute to the reduction of FSB and early deaths. ${ }^{11}$

Auscultation with the fetal stethoscope may be uncomfortable to the patient and midwives, ${ }^{6,12}$ but it is often the only method of fetal monitoring available in many units in LIC. ${ }^{6}$ Handheld Doppler devices are simple to use and relatively cheap, compared to electronic fetal monitors, and cause less maternal discomfort than the Pinard fetal stethoscope. ${ }^{13}$ On the other hand, they require electricity or batteries. ${ }^{2}$ The Freeplay wind-up handheld fetal Doppler has rechargeable batteries and can also be hand-cranked to provide rapid recharging with 1 minute of winding, providing 10 minutes of use. Its readings are reliable, and the device is well accepted by mothers and health care providers in LIC. ${ }^{14,15}$

A recent Cochrane Systematic review reported on a paucity of studies (trials) comparing intermittent auscultation of fetal heart rate in labor for fetal well-being using the methods described in this manuscript which are frequently used in low-income settings. ${ }^{16}$ Only 2 studies were identified in the subject area, and several important outcomes were not reported, indicating presence of uncertainties regarding the use of intermittent auscultation of FHR in labor. The review recommended more randomized trials in low-income settings comparing different monitoring tools and timing for intermittent auscultation.

The aim of this study was to compare the effectiveness of 2 devices for intermittent FHR monitoring during labor, ie, the Pinard fetal stethoscope (Pinard) versus the FreePlay wind-up handheld Doppler (Doppler) (Power-free Education and Technology, Cape Town, South Africa) regarding their ability to detect FHR abnormalities. Secondary outcomes were time intervals from abnormal FHR detection to delivery, mode of delivery, and perinatal outcomes (FSBs, 24-hour neonatal admissions, and deaths).

\section{Materials and methods Design}

This is a prospective nonblinded randomized controlled study comparing Pinard and Doppler for intermittent FHR monitoring. Women were randomly allocated to one of the 2 study arms by choosing Sequentially Numbered Opaque Sealed Envelopes scheme.

\section{Study setting}

This study was conducted at Muhimbili National Hospital (MNH) in Dar es Salaam from April 2013 to September 2015. MNH is a teaching hospital for the Muhimbili University of Health and Allied Sciences and is the largest consultant hospital in the United Republic of Tanzania. It is situated in Dar es Salaam, which has a population of nearly 5 million and an annual population growth rate of $4.3 \% \cdot{ }^{17}$ About 10,000 deliveries are conducted annually, corresponding to about 35 deliveries per day. The hospital serves as a tertiary referral hospital of the city and the neighboring regions. It deals with many complicated obstetric cases, $50 \%$ of these ending in cesarean section (the highest in the country). The high rate of cesarean sections is due to increased referral of complicated cases from the lower-level facilities and suboptimal indications. ${ }^{18,19}$ Deliveries are conducted by nurse-midwives and doctors, assisted by medical and midwifery students from the university.

\section{Study population}

This study involved low-risk pregnancies that met the following eligibility criteria: gestation age $\geq 37$ weeks, cephalic presentation, normal FHR on admission, and cervical dilatation $\leq 7 \mathrm{~cm}$. Exclusion criteria included women presenting with placental abruption, ruptured uterus, elective cesarean section, and multiple pregnancies. In addition, women admitted without FHR measure, or severely ill patients who could not give consent, were excluded from the study, but were managed according to the hospital protocol.

\section{Training and FHR monitoring}

Before start of the study, a 1-day workshop was conducted to train midwives and the doctors on all aspects of the research protocol as well as the detection and interpretation of FHR abnormalities, using both devices. They were trained to follow the World Health Organization guideline of monitoring FHR every 30 minutes during the first stage, and every 5-15 minutes during the second stage of labor. Midwives were trained to listen to the FHR during the last 10 minutes of every half hour, particularly before, during, 
and immediately after a contraction. Any FHR abnormalities were to be reported to the doctor on call for consideration and potential actions.

\section{Data collection and management}

Data were collected using a structured data collection form. Gestation age (GA) was based on first trimester ultrasound (if available) and self-report of the last normal menstrual period. Preterm was defined as a GA $<37$ weeks; term pregnancy was defined as $\geq 37$ and $<42$ weeks; and postterm as a GA $>42$ weeks. Maternal infection was recorded from Antenatal Cards or if the mother had any history of infection during her pregnancy. Birth weight in grams was recorded immediately after delivery using a calibrated scale in the labor ward and was dichotomized as low birth weight if $<2,500 \mathrm{~g}$ and normal if $>2,500 \mathrm{~g} .{ }^{20} \mathrm{FSB}$ was defined as an Apgar score of zero at both 1 and 5 minutes with intact skin and suspected death during labor/delivery. Antepartum death/ macerated stillbirth was defined as an Apgar score of zero at both 1 and 5 minutes with desquamated skin and suspected death before start of labor. Adverse perinatal outcomes, such as FSB, 24-hour, and admissions to neonatal unit for treatment were used as markers of suboptimal intrapartum care. A composite perinatal outcome measure included FSB, admissions, and deaths within 24 hours.

Data were double-entered in Epidata (EpiData Association, Odense, Denmark) by 2 independent data clerks. Random, periodical cross-checks were conducted on the entered data. If there were any discrepancies between the 2 entered databases, the data clerks rechecked the original data together and corrected where necessary.

\section{Outcomes}

The primary outcome measure was the detection of an abnormal baseline FHR. FHR was defined as normal if it was between 120 and 160 beats/min, and abnormal if $<120$ or $>160$ beats $/ \mathrm{min}$. Secondary outcomes included mode of delivery, time intervals during labor to delivery, newborn characteristics (ie, Apgar scores at 5 minutes and attempted bag mask ventilation), and perinatal outcome (ie, FSB and admission to neonatal unit, or death within 24 hours postpartum).

\section{Sample size calculation and statistics}

Data from another study in rural Tanzania revealed an abnormal FHR detection rate of $2.7 \%$ among low-risk deliveries using fetal stethoscopes. ${ }^{11}$ We postulated that the use of
Doppler as opposed to the Pinard would detect a minimum of 5\% abnormal FHR. To detect the differences at a significance level of 0.05 with $80 \%$ power, 1,176 women were needed in each arm, giving a total sample of 2,352 using Openepi software. ${ }^{21}$ We included 2,844 women, 20\% more than the calculated sample, to compensate for potentially missing data.

Analysis was performed with Statistical Package for Social Sciences (SPSS) 23 (IBM Corporation, Armonk, NY, USA). $\chi^{2}$ and Fisher's exact tests were used to compare proportions between groups, whereas Mann-Whitney tests and Independent sample $t$-tests were used to compare groups with respect to continuous variables. Furthermore, we report adjusted odds ratios from logistic regression analysis with $95 \%$ confidence intervals. A $p$-value of $<5 \%$ was considered statistically significant.

\section{Ethical considerations}

All women provided written informed consent to participate in and allow for publication of data before enrollment. They were informed about the study and those found to have an abnormal FHR would be managed according to hospital protocols. The trial was registered on the ClinicalTrial.gov website with identifier number NCT01869582. Ethical clearance to conduct and publish the study was given by the Publication and Ethical Committee of the Muhimbili University of Health and Allied Sciences (reference number: MU/DRP/ AEC/Vol.XVIII/105).

\section{Results}

During the study period, 20,848 women delivered at MNH, and 3,317 were eligible for recruitment (Figure 1). Of these, 2,844 (86\%) consented to participate and were randomized to either the Pinard arm $(n=1,423)$ or Doppler arm $(n=1,421)$.

Table 1 compares antenatal characteristics between the two groups. Maternal infections were significantly more common in the Doppler group ( $p=0.027$ ). There were more referred patients/inpatients in the Pinard group as compared to the Doppler group. Other parameters, such as GA, antenatal problems, and birth weight, were similar between groups (Table 1).

A comparison of primary and secondary outcomes in the two arms is presented in Table 2. Adjusted odds ratios are presented for all the variables after controlling for imbalances in the maternal variables (Table 1). There was a significantly higher proportion of FHR abnormalities detected in the Doppler (6.0\%) compared to the Pinard (3.9\%) group 


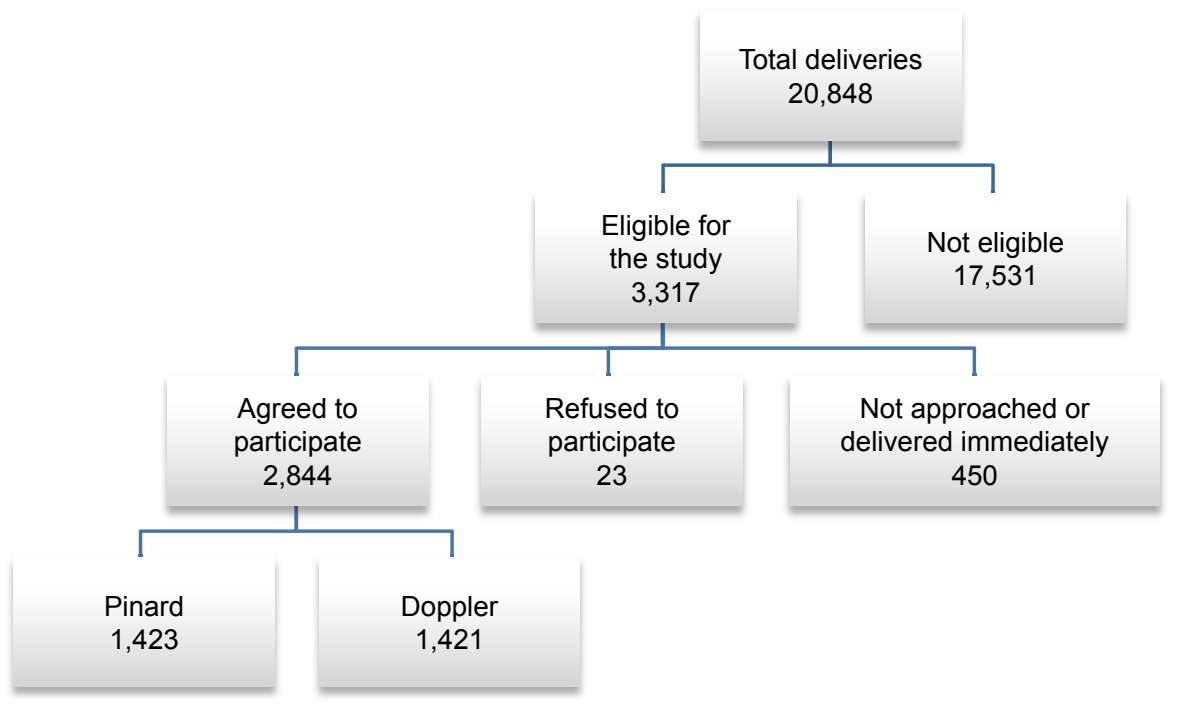

Figure I Trial profile.

(adjusted odds ratio $=1.59, p=0.008$ ). Overall, there were no significant differences in any of the secondary outcomes (Apgar score $<7$, delivery of bag mask ventilation, mode of delivery, perinatal admissions, and deaths).

The mean $( \pm \mathrm{SD})$ duration of first stage of labor was similar in both groups (ie, nearly 11 hours 30 minutes \pm 2 hours 40 minutes, $p=0.83$ ). The mean duration of second stage was slightly longer in the Doppler ( $34 \pm 14$ minutes) compared to the Pinard ( $32 \pm 14$ minutes) group ( $t$-test, $p=0.039)$. The median (interquartile range) time intervals from detection of an abnormal FHR to delivery were almost comparable, ie, $80(60,161)$ minutes in the Doppler group, and $89(52,165)$ minutes in the Pinard group (Mann-Whitney test, $p=0.88$ ) for all modes of deliveries.

Figure 2 shows that, overall, 142 (5.0\%) cases of abnormal FHR were detected in this study. Among the 16 perinatal deaths, $8(50 \%)$ were noted to have an abnormal FHR pattern. One perinatal death was recorded among newborns delivered by cesarean section. Subgroup analysis of the composite perinatal outcomes (ie, FSB and 24-hour deaths and admissions to a neonatal area) revealed that newborns with abnormal
FHR delivered vaginally had a more unfavorable outcome in the Pinard group (10 of $23 ; 43.5 \%$ ) compared to the Doppler group ( 7 of $43 ; 16.3 \%$ ) (Fisher's exact test, $p=0.021$ ). There was no time difference in this subgroup analysis between the study arms $(p=0.305)$.

\section{Discussion}

We found a higher likelihood of detecting abnormal FHR by intermittent monitoring using the Doppler technique as opposed to the Pinard. However, overall, perinatal outcomes were similar, although subgroup analysis revealed that newborns with abnormal FHR delivered vaginally had better perinatal outcomes in the Doppler compared to the Pinard group. The time intervals from detection of an abnormal FHR to delivery were long in both groups.

Our findings on FHR abnormalities are comparable to prior studies completed in Kampala and Harare, where the Doppler detected more FHR abnormalities than the Pinard fetal stethoscope. ${ }^{22,23}$ The Kampala study reported that despite a higher detection of FHR abnormalities with the Doppler technique, no improvement in perinatal outcome was seen,

Table I Comparison of maternal characteristics in the Pinard and Doppler groups among low-risk parturient women at MNH

\begin{tabular}{lllll}
\hline Antenatal characteristics & Pinard $\mathbf{n}=\mathbf{I , 4 2 3}(\%)$ & Doppler $\mathbf{n = I , 4 2 ~ I ~ ( \% ) ~}$ & Total (\%) $\mathbf{n = 2 , 8 4 4}(\%)$ & $\mathbf{p}$-value* \\
\hline Maternal infection & $28(2.0)$ & $48(3.3)$ & $76(2.7)$ & 0.027 \\
Low birth weight & $65(4.6)$ & $62(4.3)$ & $127(4.4)$ & 0.790 \\
GA $\geq 42$ weeks & $6(0.4)$ & $8(0.6)$ & $14(0.5)$ & 0.790 \\
Antenatal problem & $128(9.0)$ & $11 I(7.7)$ & $239(8.4)$ & 0.250 \\
Referred patient/inpatient & $205(14.4)$ & $158(11.0)$ & $363(12.7)$ & 0.008 \\
\hline
\end{tabular}

Note: $* \chi^{2}$ tests.

Abbreviations: GA, gestational age; $\mathrm{MNH}$, Muhimbili National Hospital. 
Table 2 Frequencies of abnormal FHR detections, newborn characteristics, and perinatal outcomes in the Pinard and Doppler arms among low-risk parturient women at $\mathrm{MNH}$

\begin{tabular}{|c|c|c|c|c|c|c|}
\hline Primary/secondary outcomes & $\begin{array}{l}\text { Pinard } \\
n=1,423(\%)\end{array}$ & $\begin{array}{l}\text { Doppler } \\
n=I, 42 \mid \text { (\%) }\end{array}$ & Unadjusted OR & $p$-value* & AOR** & $p$-value* \\
\hline Abnormality of FHR & $56(3.9)$ & $86(6.0)$ & $1.56(I .12-2.2 I)$ & 0.012 & $1.59(1.13-2.26)$ & 0.008 \\
\hline Mode of delivery: cesarean section & $174(12.2)$ & $172(12.0)$ & $0.98(0.79-1.23)$ & 0.89 & $0.96(0.77-1.21)$ & 0.76 \\
\hline Apgar 5 minutes $<7$ & $23(1.6)$ & $30(2.1)$ & I.3I $(0.76-2.26)$ & 0.40 & $1.38(0.79-2.24)$ & 0.25 \\
\hline Bag mask ventilation attempted & $68(4.8)$ & $76(5.3)$ & $1.19(0.80-1.57)$ & 0.51 & $1.18(0.84-1.65)$ & 0.35 \\
\hline Admissions to neonatal unit at birth & $28(2.0)$ & $38(2.7)$ & $1.36(0.82-2.25)$ & 0.24 & $1.42(0.86-2.33)$ & 0.17 \\
\hline Fresh stillbirths & $8(0.6)$ & $5(0.3)$ & $0.63(0.20-1.92)$ & 0.41 & $0.67(0.22-2.07)$ & 0.49 \\
\hline Still admitted at 24 hours & $18(1.3)$ & $22(1.5)$ & $1.22(0.65-2.28)$ & 0.63 & $1.25(0.66-2.34)$ & 0.49 \\
\hline $\begin{array}{l}\text { Perinatal deaths (FSB + deaths } \\
\text { within } 24 \text { hours) }\end{array}$ & $10(0.7)$ & $6(0.4)$ & $0.59(0.22-1.65)$ & 0.32 & $0.62(0.26-1.73)$ & 0.36 \\
\hline $\begin{array}{l}\text { Composite outcomes (perinatal } \\
\text { deaths and admissions) }\end{array}$ & $28(2.0)$ & $28(2.0)$ & $0.99(0.59-1.69)$ & 0.98 & $0.73(0.34-\mathrm{I} .47)$ & 0.35 \\
\hline
\end{tabular}

Notes: Data are presented as $\mathrm{n}(\%)$ and AOR. *Wald test. $\chi^{2}$ test. **Multiple logistic regression analysis adjusted for maternal infection and sources of admission. Abbreviations: AOR, adjusted odds ratio; FHR, fetal heart rate; FSB, fresh stillbirth; MNH, Muhimbili National Hospital.

which is similar to our overall finding. A suggested reason for this relates to several contextual constraints in low-resource settings leading to lack of timely interventions to deliver the baby. ${ }^{9}$ These constraints include, among others, a delay in decision-making due to a high patient to staff ratio in the labor ward, and often multiple simultaneous patients waiting for an emergency cesarean section. ${ }^{18,22}$ A longer than recommended decision-to-delivery time interval may in part explain the lack of difference in perinatal outcomes between the groups in the present study. One would have anticipated that the higher detection rate of abnormal FHR by Doppler would lead to a timelier intervention such as a cesarean section. However, the frequency of a cesarean section was unaffected, and we speculate that the striking imbalance between available health resources (staff, access to theater) and large volume of patients likely play a crucial role. ${ }^{24,25}$ Therefore, in order to effectively manage critical cases and improve perinatal outcomes, improved FHR monitoring techniques coupled with better staffing, as well as improved equipment and theater facilities, ie, an overall increased capacity and improved systems, are necessary in order to affect perinatal outcomes.

A subgroup analysis of those newborns with a detected abnormal FHR, delivered vaginally, revealed improved composite perinatal outcomes in the Doppler compared to the Pinard group. These findings are similar to those found in the Harare study, ${ }^{21}$ which involved dedicated research midwives, and where improved perinatal outcomes were reported in the Doppler arm. These findings may also indicate that midwives can detect FHR abnormalities more frequently and earlier when using the Doppler technique compared to a fetal stethoscope, thereby recognizing signs of intrapartum hypoxia more often and at an earlier stage.
There might be several reasons for more and/or earlier detection of abnormal FHR in the Doppler group. The Doppler technique provides digital sound and readings, which do not require much skill to interpret and can easily be confirmed by peers, as opposed to the Pinard, which requires a complete minute of counting. ${ }^{15}$ Additionally, midwives may feel unsure about the reliability of the Pinard assessments..$^{18}$ A qualitative study (in-depth interviews and focus group discussions) performed among labor ward staff at $\mathrm{MNH}$ reports that the Doppler was the preferred device for improving FHR monitoring. ${ }^{26}$

\section{Limitations}

This study was not powered to detect overall differences in perinatal outcomes. A study involving an appropriate sample size, perhaps in a more high-risk population and coupled with timely obstetric intervention, might be able to show differences in overall perinatal outcomes. Second, the documented fear of blame from peers and hospital management at $\mathrm{MNH}$, as suggested in the qualitative study previously conducted in the same hospital, ${ }^{26}$ might have led to a defensive practice with overreporting of abnormal FHR. Third, in this study, we have not been able to perform other tests, such as fetal scalp $\mathrm{pH}$ levels, to confirm possible fetal hypoxic state. Fourth, the study involved 2 different medical devices in measuring FHR, and it was not possible to blind the patients and providers.

\section{Conclusion and recommendation}

Monitoring of FHR using a wind-up Doppler was associated with an increased detection of abnormal FHR. Overall perinatal outcomes were comparable between groups, but there were better perinatal outcomes for newborns with detected abnormal FHR delivered vaginally in the Doppler 


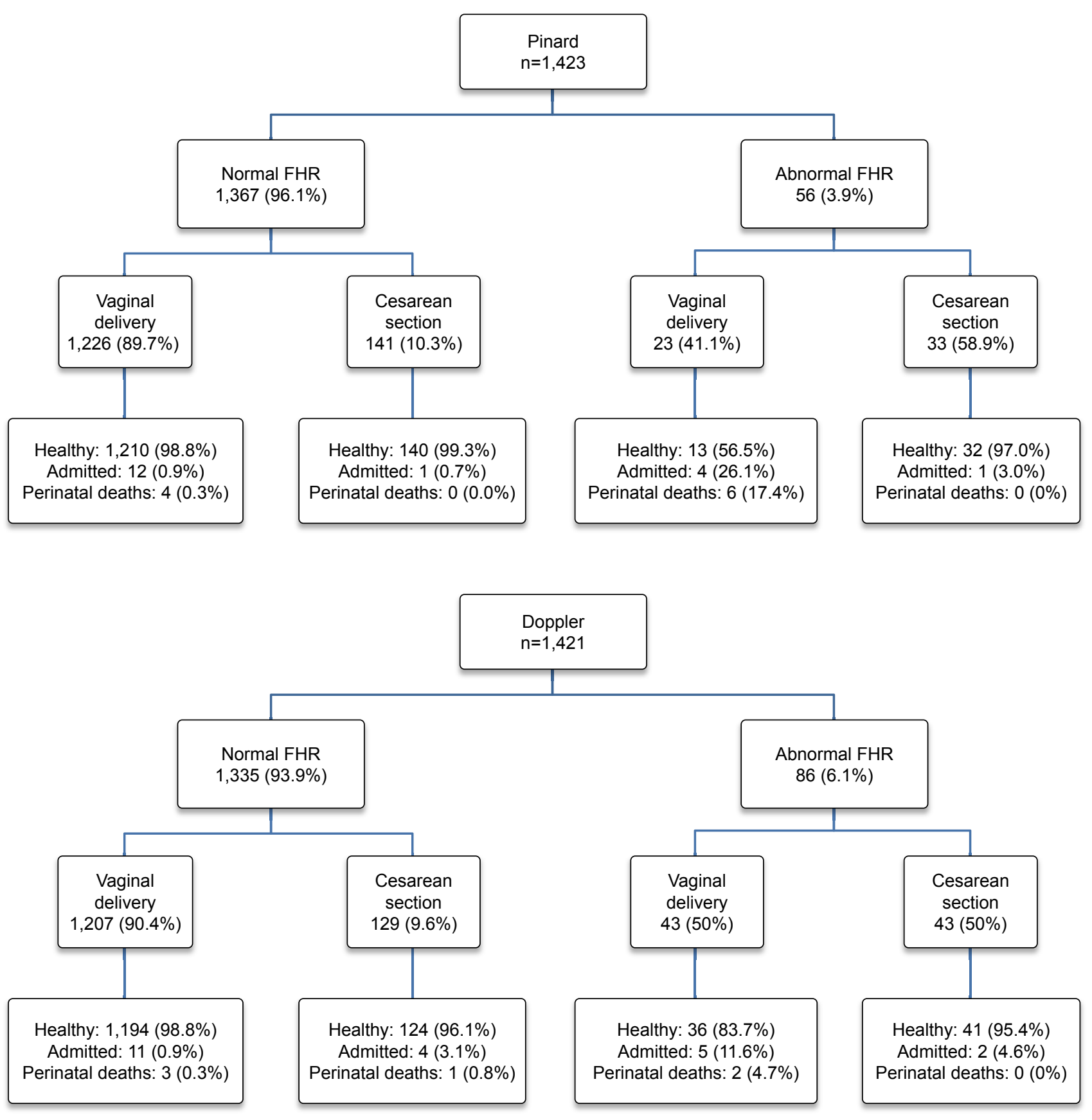

Figure 2 Flow diagrams of FHR detections, mode of delivery, and perinatal outcomes in both arms, Pinard and Doppler. Abbreviation: FHR, fetal heart rate.

group. A study powered for perinatal outcomes, coupled with timely interventions, may be able to demonstrate differences in overall perinatal outcome.

\section{Data sharing statement}

Data can be made available to editors and/or reviewers upon request from Research, Teaching and Consultancy Unit of Muhimbili National Hospital (address: PO Box 65000, Dar es Salaam, Tanzania; phone: +255 22215 1599; fax: +255 22215 0534; email: info@mnh.or.tz)

\section{Acknowledgment}

We thank the hospital administration for allowing us to use the hospital facilities to collect data for this study. We also wish to acknowledge the entire midwife team for the excellent job of keeping records of all deliveries in the midwifery book that was then computerized. We thank the well-motivated data clerks of the Safer Births project for data entry and cleaning. The Laerdal Foundation funded the study. The funder had no role in the study design, data collection, analysis, or decision to publish or preparation of the manuscript. 


\section{Author contributions}

BAK conducted the initial statistical data analysis and prepared all sections of the first draft and compiled the final manuscript. HLK conceived and designed the study and supervised the training of research midwives and the data collection process. He was also involved in data analysis and the interpretation of the results. PJW and ERM participated in the design of the study and the review of the manuscript. ID reviewed the statistical analysis and the final draft of the manuscript. HLE and JMP were involved in design of the study, interpretation of data, and critically reviewed the manuscript. All authors contributed toward data analysis, drafting and critically revising the paper and agree to be accountable for all aspects of the work. All authors agreed on the final submitted manuscript.

\section{Disclosure}

BAK received unconditional funding for studies from Laerdal Foundation. The other authors report no conflicts of interest in this work.

\section{References}

1. Lawn JE, Lee AC, Kinney M, et al. Two million intrapartum-related stillbirths and neonatal deaths: where, why, and what can be done? Int J Gynaecol Obstet. 2009;107(Suppl 1):S5-S18, S19.

2. Wyatt J. Appropriate medical technology for perinatal care in lowresource countries. Ann Trop Paediatr. 2008;28(4):243-251.

3. Lawn J, Shibuya K, Stein C. No cry at birth: global estimates of intrapartum stillbirths and intrapartum-related neonatal deaths. Bull World Health Organ. 2005;83:409-417.

4. Hill K, Thomas K, AbouZahr C, et al. Estimates of maternal mortality worldwide between 1990 and 2005: an assessment of available data. Lancet. 2007;370(9595):1311-1319.

5. UNICEF, WHO, World Bank Group and United Nations. Levels and Trends in Child Mortality Child Mortality. New York, NY: UNICEF; 2017.

6. Martis R, Emilia O, Nurdiati DS, Brown J. Cochrane Database of Systematic Reviews. Martis R, editor. Vol 2. Hoboken, NJ: John Wiley \& Sons, Ltd; 2017:CD008680.

7. Spencer-Jones J. Make every mother and child count. S Afr Med J. 2005;95(6):382, 384.

8. van Handel M, Swaab H, de Vries LS, Jongmans MJ. Long-term cognitive and behavioral consequences of neonatal encephalopathy following perinatal asphyxia: a review. Eur J Pediatr. 2007;166(7):645-654.

9. Maaløe N, Housseine N, Meguid T, et al. Effect of locally tailored labour management guidelines on intrahospital stillbirths and birth asphyxia at the referral hospital of Zanzibar: a quasi-experimental pre-post study (The PartoMa study). BJOG An Int J Obstet Gynaecol. 2018;125(2):235-245.

10. WHO and UNICEF. Every Newborn: An Action Plan to End Preventable Deaths. Geneva: World Health Organization; 2014.
11. Langli Ersdal H, Mduma E, Svensen E, Sundby J, Perlman J. Intermittent detection of fetal heart rate abnormalities identify infants at greatest risk for fresh stillbirths, birth asphyxia, neonatal resuscitation, and early neonatal deaths in a limited-resource setting: a prospective descriptive observational study at Haydom Lutheran Hospital. Neonatology. 2012;102:235-242.

12. Wyatt J. Appropriate medical technology for perinatal care in lowresource countries. Ann Trop Paediatr. 2008;28(4):243-251.

13. Vintzileos AM, Antsaklis A, Varvarigos I, Papas C, Sofatzis I, Montgomery JT. A randomized trial of intrapartum electronic fetal heart rate monitoring versus intermittent auscultation. Obstet Gynecol. 1993;81(6):899-907.

14. Woods D. Appropriate technology and education for improved intrapartum care in underresourced countries. S Afr J Obstet Gynaecol. 2009;15(3):78-79.

15. Bezuidenhout H, Woods D, Wyatt J, Lawn J. Does fetal heart rate count? Developing a low cost, alternative powered Doppler fetal heart monitor for use in low resource high mortality settings. In: 4th IET Seminar on Appropriate Healthcare Technologies for Developing Countries; 2006:155-159; London.

16. Martis R, Emilia O, Nurdiati DS, Brown J. Cochrane Database of Systematic Reviews. Martis R, editor. Hoboken, NJ: John Wiley \& Sons, Ltd; 2017.

17. National Bureau of Statistics (NBS) and Office of Chief Government Statistician (OCGS), Zanzibar. The 2012 Population and Housing Census: Basic Demographic and Socio-Economic Profile; Key Findings. Dar es Salaam, Tanzania: NBS and OCGS; 2014. Available from: http:// nbs.go.tz/nbs/takwimu/census2012/Basic_Demographic_and_SocioEconomic_Profile_PopularVersion-KeyFindings_2012_PHC_EnglishVersion.pdf. Accessed November 11, 2015.

18. Mgaya AH, Litorp H, Kidanto HL, Nyström L, Essén B. Criteria-based audit to improve quality of care of foetal distress: standardising obstetric care at a national referral hospital in a low resource setting, Tanzania. BMC Pregnancy Childbirth. 2016;16(1):343.

19. Litorp H, Kidanto HL, Nystrom L, Darj E, Essén B. Increasing caesarean section rates among low-risk groups: a panel study classifying deliveries according to Robson at a university hospital in Tanzania. BMC Pregnancy Childbirth. 2013;13(1):107.

20. World Health Organization. International Statistical Classification of Diseases and Related Health Problems, Tenth Revision. Geneva: World Health Organization; 2011;18:39.

21. Kelsey J, Whittemore A, Evans A, Thompson D. Methods in Observational Epidemiology. Oxford: Oxford University Press; 1996.

22. Byaruhanga R, Bassani DG, Jagau A, Muwanguzi P, Montgomery AL, Lawn JE. Use of wind-up fetal Doppler versus Pinard for fetal heart rate intermittent monitoring in labour: a randomised clinical trial. $B M J$ Open. 2015;5(1):e006867.

23. Mahomed K, Nyoni R, Mulambo T, Kasule J, Jacobus E. Randomised controlled trial of intrapartum fetal heart rate monitoring. BMJ. 1994;308(6927):497-500.

24. Shemdoe A, Mbaruku G, Dilip A, et al. Explaining retention of healthcare workers in Tanzania: moving on, coming to "look, see and go," or stay? Hum Resour Health. 2016;14:2.

25.The United republic of Tanzania Ministry of Health and Social Welfare. Human Resource for Health and Social Welfare Strategic Plan 2014-2019. Dar es Salaam, Tanzania: Ministry of Health and Social Welfare; 2014.

26. Litorp H, Mgaya A, Mbekenga CK, et al. Fear, blame and transparency: obstetric caregivers' rationales for high caesarean section rates in a low-resource setting. Soc Sci Med. 2015;143:232-240. 
International Journal of Women's Health

Dovepress

\section{Publish your work in this journal}

The International Journal of Women's Health is an international, peerreviewed open-access journal publishing original research, reports, editorials, reviews and commentaries on all aspects of women's healthcare including gynecology, obstetrics, and breast cancer. The manuscript management system is completely online and includes

a very quick and fair peer-review system, which is all easy to use. Visit http://www.dovepress.com/testimonials.php to read real quotes from published authors.

Submit your manuscript here: http://www.dovepress.com/international-journal-of-womens-health-journal 